\title{
Analysis of PSPHL as a candidate gene influencing the racial disparity in endometrial cancer
}

\author{
Jay E. Allard ${ }^{1}$, Gadisetti V. R. Chandramouli ${ }^{2}$, Katherine Stagliano ${ }^{3}$, Brian L. Hood ${ }^{4}$, Tracy Litzi ${ }^{1,4}$, \\ Yutaka Shoji ${ }^{2,3}$, Jeff Boyd ${ }^{3,5}$, Andrew Berchuck ${ }^{6}$, Thomas P. Conrads ${ }^{3}$, G. Larry Maxwell ${ }^{1,4}$ and \\ John I. Risinger ${ }^{2,3 *}$ \\ ${ }^{1}$ Walter Reed Army Medical Center, Washington, DC, USA \\ 2 Department of Obstetrics, Gynecology and Reproductive Biology, Michigan State University College of Human Medicine, Grand Rapids, MI, USA \\ ${ }^{3}$ Curtis and Elizabeth Anderson Cancer Institute at Memorial Health University Medical Center, Savannah, GA, USA \\ ${ }^{4}$ Women's Health Integrated Research Center at Inova Health System, Annandale, VA, USA \\ ${ }^{5}$ Fox Chase Cancer Center, Philadelphia, PA, USA \\ ${ }^{6}$ Division of Gynecologic Oncology, Duke University, Durham, NC, USA
}

\section{Edited by:}

Angeles Alvarez Secord, Duke University Medical Center, USA

Reviewed by:

Miriam Reuschenbach, University Hospital Heidelberg, Germany

Cara Mathews, University of

Oklahoma Health Sciences Center,

USA

\section{*Correspondence:}

John I. Risinger, Department of

Obstetrics, Gynecology and

Reproductive Biology, Van Andel Institute, Michigan State University

College of Human Medicine, 333

Bostwick Avenue, Room 4019, Grand

Rapids, MI 49503, USA.

e-mail: john.risinger@hc.msu.edu

\begin{abstract}
Endometrial cancer is the most commonly diagnosed gynecologic malignancy in the United States. A well recognized disparity by race in both incidence and survival outcome exists for this cancer. Specifically Caucasians are about two times more likely to develop endometrial cancer than are African-Americans. However, African-American women are more likely to die from this disease than are Caucasians. The basis for this disparity remains unknown. Previous studies have identified differences in the types and frequencies of gene mutations among endometrial cancers from Caucasians and African-Americans suggesting that the tumors from these two groups might have differing underlying genetic defects. We performed a gene expression microarray study in an effort to identify differentially expressed transcripts between African-American and Caucasian women's endometrial cancers. Our gene expression screen identified a list of potential biomarkers that are differentially expressed between these two groups of cancers. Of these we identified a poorly characterized transcript with a region of homology to phospho serine phosphatase (PSPH) and designated phospho serine phosphatase like (PSPHL) as the most differentially overexpressed gene in cancers from African-Americans. We further clarified the nature of expressed transcripts. Northern blot analysis confirmed the message was limited to a transcript of under $1 \mathrm{kB}$. Sequence analysis of transcripts confirmed two alternate open reading frame (ORF) isoforms due to alternative splicing events. Splice specific primer sets confirmed both isoforms were differentially expressed in tissues from Caucasians and African-Americans. We further examined the expression in other tissues from women to include normal endometrium, normal and malignant ovary. In all cases PSPHL expression was more often present in tissues from African-Americans than Caucasians. Our data confirm the African-American based expression of the PSPHL transcript in endometrial cancer and also identify its expression in other tissues from African-Americans including ovary and ovarian cancer. PSPHL represents a candidate gene that might influence the observed racial disparity in endometrial and other cancers.
\end{abstract}

Keywords: endometrial cancer, PSPHL, racial disparity

\section{INTRODUCTION}

A racial disparity in incidence and survival exists for many human cancers. Understanding and addressing the reasons for these disparities is critical for reducing cancer burdens. A well described disparity in both incidence and survival outcome exists for endometrial cancer. Population based studies using data from both the National Cancer Data Base and Surveillance, Epidemiology, and End Results (SEER) have consistently shown that Caucasians (CA) are more likely to develop this cancer than are African-Americans (AA) even after controlling for hysterectomy (Hicks et al., 1998; Sherman and Devesa, 2003; Sherman et al., 2005). However, African-American women are about two times more likely to die from their disease than are Caucasians (Hicks et al., 1998; Ghafoor et al., 2002). The reasons underlying these disparities are complex and include social, cultural and biologic factors (Maxwell and Risinger, 2006). Several molecular genetic studies have identified differences in the prevalence of certain gene mutations or expression aberrations in cancers from $\mathrm{AA}$ and CA women. For example, the TP53 tumor suppressor gene is mutated more frequently in endometrial cancers from AA women (Kohler et al., 1996). Similarly the HER2/Neu oncogene is more frequently up-regulated in endometrial cancer from AA women (Santin et al., 2005). In addition, a specific chromosome gain on chromosome one is more frequent in endometrial cancers from 
AA women (Morrison et al., 2010). Epigenetic methylation of the ribosomal DNA is also less prevalent in endometrial cancers from AA women (Powell et al., 2002). TP53, rDNA, and HER2 events are tied to more aggressive behavior and adverse outcome in endometrial cancer, suggesting they may be responsible in part for the outcomes disparity for AA women. In contrast, the PTEN tumor suppressor gene is more frequently mutated in cancers from $\mathrm{CA}$ and is associated with a more favorable outcome (Maxwell et al., 2000). It is important to note that African-Americans more often have non-endometrioid cancers and the above referenced gene changes are often more frequently associated with specific histotype e.g., TP53 and serous and PTEN with endometrioid type (Kohler et al., 1996; Maxwell et al., 2000; Wright et al., 2009).

Recently, we performed transcript expression studies in endometrial cancers (Risinger et al., 2003, 2005; Maxwell et al., 2005; Ferguson et al., 2006). These studies showed distinct expression related to histologic type as well as identifying transcripts associated with the microsatellite instability phenotype. In this study we specifically compared stage and grade matched endometrial cancers from AA and CA patients to identify whether distinct transcripts were associated with the race of the patient and, if so, whether these could serve as candidates for diagnosis, prognosis, or intervention. A previous study using a very similar study design found no global differences between AA and CA but did identify a small sub-set of genes differentially expressed. Although global differences between the two groups are not evident, we do identify a number of statistically differentially expressed transcripts, a subset of which were validated by quantitative real time PCR. Among those validated was that encoded by the gene for phosphoserine phosphatase like (PSPHL), which was found to be prominently expressed in endometrial cancer specimens from AA women and cloned an additional splice variant also preferentially expressed in AA women.

\section{RESULTS \\ GENE EXPRESSION OF AFRICAN-AMERICAN AND CAUCASIAN ENDOMETRIAL CANCERS}

We performed a hybridization-based transcript expression analysis (Affymetrix) on a set of endometrial cancer specimens that were controlled for stage and grade and examined the global transcript expression of these cancers using multidimensional scaling. Cancers representing the groups were not found to be distinct indicating that race was not the chief determinant of gene expression between these two groups (Figure 1A). Class comparison tests between AA and CA indicated 341 transcripts at $p<0.005$ with a global test $p$-value of 0.061 . We further examined the data using principal component analysis (PCA). The first two principal components (PCs) explaining highest variance did not distinguish AA and CA classes. However, a sub-set of CA cases partially segregated from AA cases along $\mathrm{PC} \# 3$ which explains only $7.2 \%$ of the total variance (Figure 1B). Despite the lack of global difference we determined which transcripts were differentially expressed between these groups by paired $t$-tests to control for histology, stage, and grade variations. We noted a total of 263 genes (325 transcripts) at $p<0.005$ (Table S1 in Supplementary Material) 66 of these marked by at least a twofold change are depicted in Figure 2.

\section{QUANTITATIVE VALIDATION OF DIFFERENTIALLY EXPRESSED TRANSCRIPTS}

Findings on gene expression microarrays require validation using alternate sample sets and more quantitative methodologies. We performed quantitative real time (qRT) PCR to validate the array findings for a sub-set of transcripts identified. Specifically we examined the Insulin-like Growth Factor Receptor 1, (IGFR1) Mucin 20, (MUC20), Ras Related Associated with Diabetes, $(R R A D)$, and the Phosphoserine Phosphatase (PSPH) genes using $\mathrm{qRT}$-PCR. Data from these assays performed on a panel of

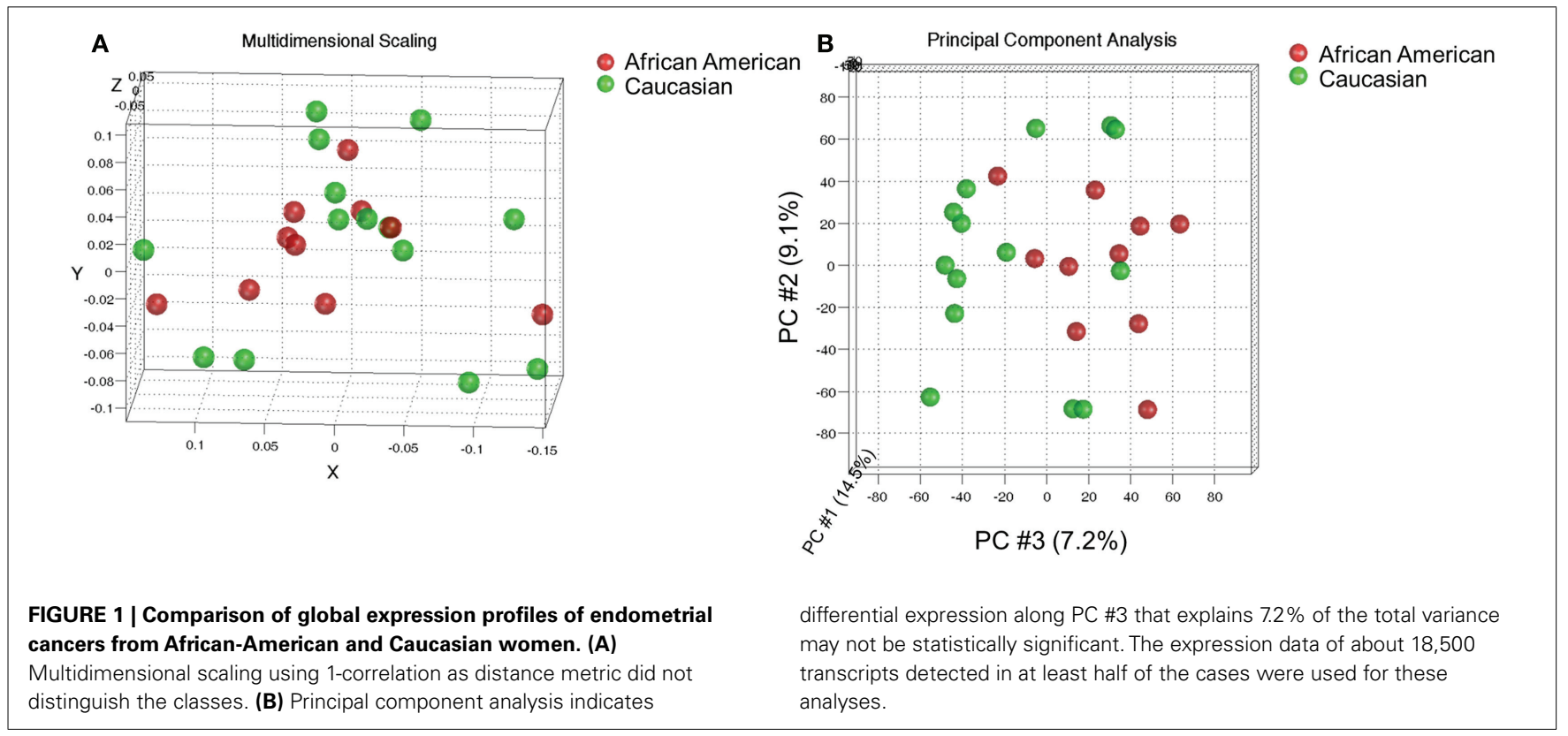




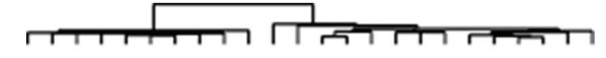

AFRICAN AMERICAN

CAUCASIAN

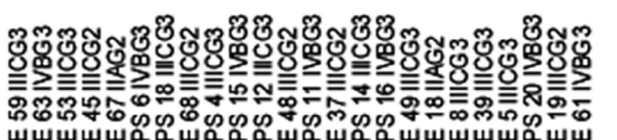

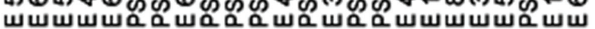

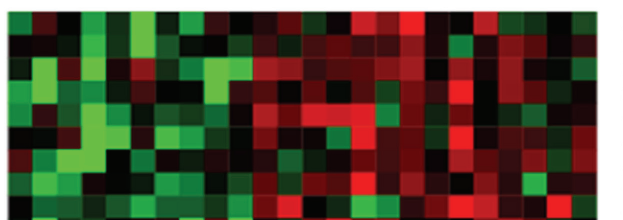

Affymetrix Id

Gene

Description

242824 at EST:HIGC15.06.00 Transcribed sequence

210061-at ZNF589 zinc finger protein 589

233366_at FBXO4 F-box only protein 4

234204_at FLل21644 fis CDNA: FLJ21644 fis, clone COL08411

myocardin

230959_at EST:wy84b12.x1 Transcribed sequences

227790-at C6orf157 chromosome 6 open reading frame 157

232382 s_at LOC115294 similar to hypothetical protein FLJ10883

215780_s_at SET SET translocation (myeloid leukemia-associated)

222048_at ADRBK2 adrenergic, beta, receptor kinase 2

$220394^{-}$at FGF20

217143_s_at TRD@

215643-at SEMÄ3D

204939_s_at PLN

204939 -s_at PLN

$212958^{-} \times$- at PAM

230543-at USP9X

237950 -s_at EST:AV649666

212206_s_at H2AFV

207826 s at ID3

206796-at WISP1

211571_s_at CSPG2

206847-s-at HOXA7

233573 - s-at WDR6

224428 s at CDCA7

243349 at KIAA1324

236610_at EST:0z48e01.x

206560_s_at MIA

218847_at IMP-2

236798-at EST:xv35c04.x

228275-at EST: $993907 \times 1$

227955 s_at EST:7e33a07 $\times 1$

$203550^{-}$- _at C1orf2

$216766^{-}{ }^{-} \bar{t}$

216766 at

232498 -at

$203628^{-a t}$ at

216828-at

205048 s at PSPH

FL21499 fis

KIAA1833

IGF1R

IGF1R

FRG1(similar)

219271_at GALNT14

209994 s at ABCB1

$204802^{-}$at RRAD

215842 s at ATP $11 \mathrm{~A}$

228919-at AST:nk67d10.s1

226322_at ARG9a

213856 at at

CD47

219327_s_at GPRC5C

231941_s at MUC2O

227253 at

$203559^{-}$s at ABP1

227943 at EST:we91d01 x1

$219008^{-}$at FLJ21820

224952-at DKFZP564D166

$227067_{-}^{-}$_at N2N

227067 $x_{-}$at N2N

219983_at

226190_at EST:602297045F1

219984_s_at HRASLS

214091_s_at GPX3

201348-at GPX3

208146 s_ at CPVL

$241703^{-a t}$ RPIBS

226021_at RDH10 fibroblast growth factor 20

$T$ cell receptor delta locus

sema domain, 3D

phospholamban

peptidylglycine alpha-amidating monooxygenase

peptidylglycine alpha-amidating monooxygenase

ubiquitin specific protease 9 , X-linked

Transcribed sequences

H2A histone family, member $V$

inhibitor of DNA binding 3

chondroitin sulfate proteoglycan 2 (versican)

homeo box A7

WD repeat domain 6

cell division cycle associated 7

maba1

Transcribed sequence

melanoma inhibitory activity

IGF-II mRNA-binding protein 2

CDNA FLJ32438 fis, clone SKMUS2001402

CDNA FLJ32438 fis, clone SKMUS2001402

CDNA: FLJ22256 fis, clone HRC02860

chromosome 1 open reading frame 2

synaptotagmin $\mathrm{V}$

Homo sapiens cDNA: FLJ21499 fis, clone COL05634.

hypothetical protein KIAA1833

insulin-like growth factor 1 receptor

insulin-like growth factor 1 receptor

novel gene similar to FRG1

phosphoserine phosphatase

proprotein convertase subtilisin/kexin type 1 inhibitor

UDP-N-acetyl-alpha-D-galactosamine

ATP-binding cassette, sub-family B, member 1

Ras-related associated with diabetes

ATPase, Class VI, type 11A

ESTs with strong similarity Cell division cycle 2-like 1

proline rich Gla (G-carboxyglutamic acid) 1

CD47 antigen

$\mathbf{G}$ protein-coupled receptor, family $\mathrm{C}$, group 5 , member $\mathrm{C}$

mucin 20

ceruloplasmin (ferroxidase)

amiloride binding protein 1

MRNA; cDNA DKFZp686A01184

hypothetical protein FLJ21820

putative ankyrin-repeat containing protein

similar to $\mathrm{NOTCH} 2$ protein

HRAS-like suppressor

CDNA clone IMAGE:4815204, partial cds

HRAS-like suppressor

glutathione peroxidase 3 (plasma)

glutathione peroxidase 3 (plasma)

carboxypeptidase, vitellogenic-like

Rap2-binding protein 9

retinol dehydrogenase 10 (all-trans)
WNT1 inducible signaling pathway protein 1

CD47 antigen

\section{$\begin{array}{llll}<1 / 4 & 1 & 4> & \text { Ratio }\end{array}$}

FIGURE 2 | Heat map of $\mathbf{6 6}$ transcripts differentially expressed between African-American and Caucasian by twofold and $\boldsymbol{p}<\mathbf{0 . 0 0 5}$. Expressions shown are mean centered. The red indicates increased and green down-regulated expression while black indicates mean value.

endometrial cancers from AA and CA confirmed the array findings for IGF1R, MUC20, and RRAD, but interestingly, qRT-PCR utilizing primers targeted to $P S P H$ did not (Figure 3). Close inspection of the (Affymetrix) probe sequence indicated that the microarray probes were actually situated in a poorly described sequence locus on the opposite arm of chromosome 7. This transcript is 


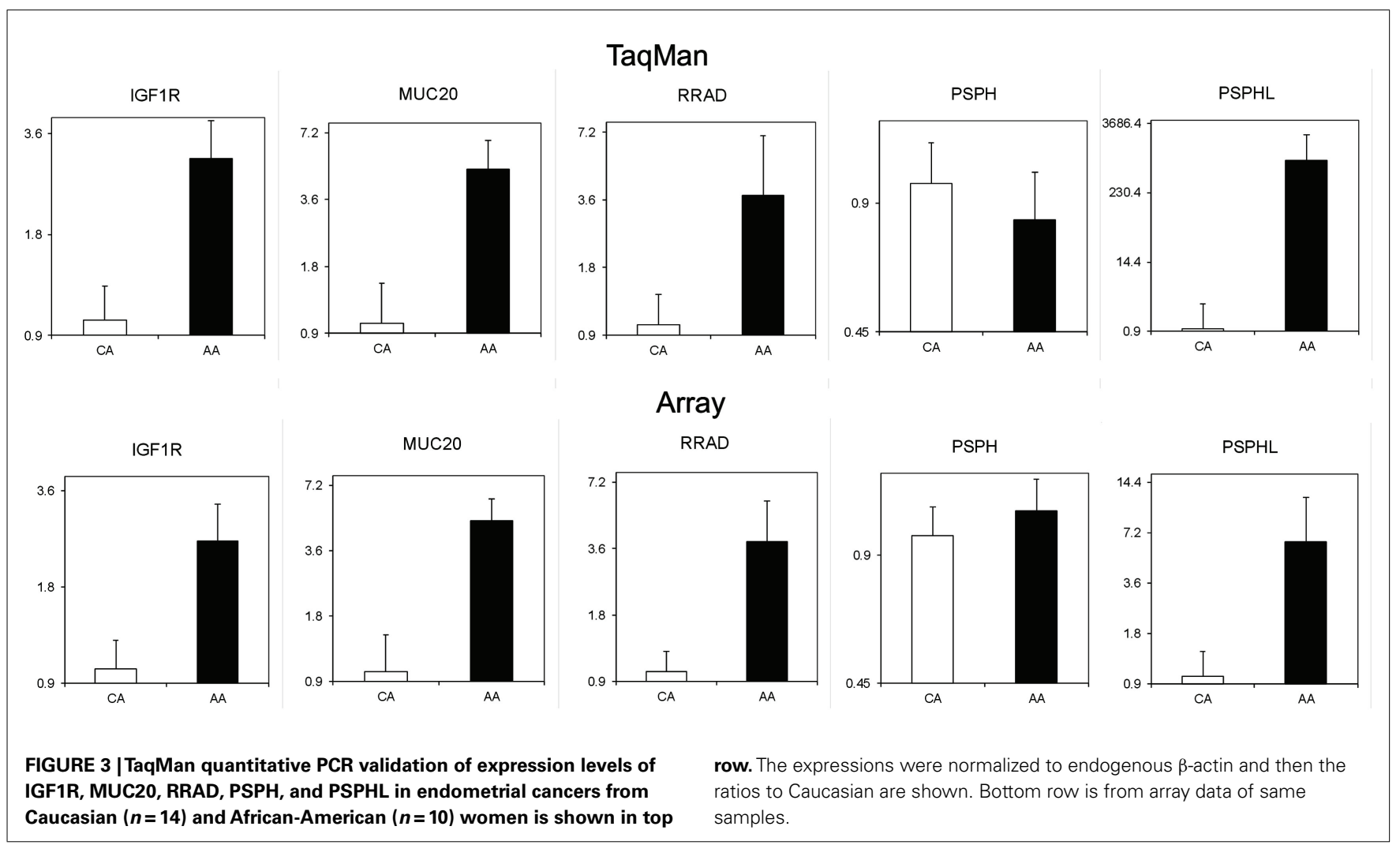

alternatively designated as CO9 or PSPHL due to sequence identity to parts of $P S P H$. This observation motivated the design of primers specific for PSPHL and re-analysis of the endometrial cancer specimens by qRT-PCR definitively demonstrates elevated expression of this transcript in AA as compared to CA patients (Figure 3).

\section{OVERLAP WITH PREVIOUS PUBLISHED MICROARRAY DATA}

We compared the differentially expressed genes with those described by Ferguson et al. (2006). We noted that probe set misannotated for PSPH was the single overlapping gene when we compared those transcripts identified at $p<0.001$ (Figure 4) This fact and the general observation that there were few genes identified and those with low $p$-values exhibit small fold differences for most genes further suggesting the effect of race on gene expression is not prominently delineated from this type of microarray analysis.

\section{TRANSCRIPTS FROM THE PSPHL GENE}

Deposited transcript information for this locus is represented by one mRNA and three spliced ESTs (BG183407, BG196884, and BX380670). In addition a publication described a related $P S P H$ sequence as the CO9 gene and published a 840 bp long sequence with homology to exons of $P S P H$ and matched the $3^{\prime}$ end of the consensus PSPHL transcript deposited in NCBI (Planitzer et al., 1998). To clarify the nature of the over-expressed sequence we amplified fetal cDNA using primers homologous to the predicted ORF of PSPHL. This PCR gave two prominent bands following

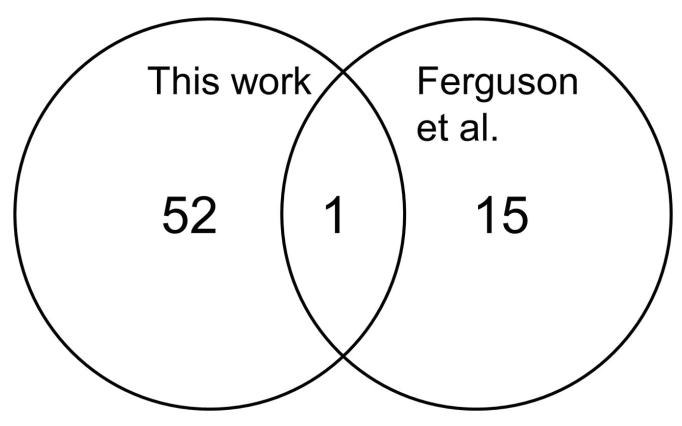

FIGURE 4 | Comparison of the numbers of differentially expressed transcripts from this work with the report of Ferguson et al. (2006). Only the transcripts altered by 1.4 -fold at $p<0.001$ between the endometrial cancers from African-American and Caucasian are shown. PSPHL is found to be highly expressed in African-American cases in both studies.

agarose gel electrophoresis (data not shown). These PCR products were cloned in the pENTR Topo vector, resultant individual colonies isolated, and plasmid clones were sequenced. We confirmed the sequence of an isoform identical to the PSPHL ORF as well as a second isoform with an apparent alternative splice that matched no other deposited RNA. These sequences contain ORFs that predict small peptides with a similar n-termini with a PSPH homologous region but differing in their c-terminal end due to frame shifting of the ORF (Figure 5). Potential peptides generated from the two longest ORF from these isoforms are very small at 72 


\section{A PSPHL-A PSPHL-B \\ PSPHL-A PSPHL-B \\ PSPHL-A PSPHL-B \\ PSPHL-A PSPHL-B \\ PSPHL-A PSPHL-B \\ PSPHL-A PSPHL-B \\ PSPHL-A PSPHL-B \\ PSPHL-A PSPHL-B \\ PSPHL-A PSPHL-B \\ PSPHL-A PSPHL-B \\ PSPHL-A PSPHL-B \\ PSPHL-A PSPHL-B \\ PSPHL-A PSPHL-B \\ PSPHL-A PSPHL-B \\ PSPHL-A PSPHL-B \\ PSPHL-A PSPHL-B \\ B}

PSPHL A protein PSPHL_B protein

PSPHL_A protein PSPHL_B protein
1 AAGCCACAGGCTCCCTGGCTGGCGTCAGCTAAAGTGGCTGTTGGGTGTCCGCAGGCTTCT

1 AAGCCACAGGCTCCCTGGCTGGCGTCAGCTAAAGTGGCTGTTGGGTGTCCGCAGGCTTCT

61 GCCTGGCCGCCGCCGCCTATAAGCTACCAGGAGGAGCTTTACGACTTCCCGTCCTGCGGG 61 GCCTGGCCGCCGCCGCCTATAAGCTACCAGGAGGAGCTTTACGACTTCCCGTCCTGCGGG

121 AAGTGGCGGGCACGATCGCAAGGTAGCGCAGAAGCTTCTCAATGGCCAGCGCCAGCTGCA 121 AAGTGGCGGGCACGATCGCAAGGTAGCGCAGAAGCTTCTCAATGGCCAGCGCCAGCTGCA

181 GCCCCGGCGGCGCACTCGCCTCACCTGAGCCTGGGAGGAAAATTCTTCCAAGGATGATCT

241 CCCACTCAGAGCTGAGGAAGCTTTTCTACTCAGCAGATGCTGTGTGTTTTGATGTTGACA 241 CCCACTCAGAGCTGAGGAAGCTTTTCTACTCAGCAGATGCTGTGTGTTTTGATGTTGACA

301 GCACGGTCATCAGTGAAGAAGGAATC-

301 GCACGGTCATCAGTGAAGAAGGAAT CGGACGGAGTCTCGCTCTGTCACCAGGCTGGAGTG 360

327

361 CAGTGGTGCGGTCTCGGCTCACTGCAACCTCCGCCTCCTGGGTTCAGGCAATTCTCCTGC

327 --GGATGCTTTCATTGGATTTGGAGGAAATGTGA

421 CTCCACCTCCTGAGTAGCTGAAACTACAGGATGCTTTCATTGGATTTGGAGGAAATGTGA

359 TCAGGCAACAAGTCAAGGATAACGCCAAATGGTATATCACTGATTTTGTAGAGCTGCTGG 481 TCAGGCAACAAGTCAAGGATAACGCCAAATGGTATATCACTGAT TTTGTAGAGCTGCTGG

419 GAGAACCGGAAGAATAACATCCATT GTCATACAGCTCCAAACAACTTCAGATGAATTTTT 541 GAGAACCGGAAGAATAACATCCATT GTCATACAGCTCCAAACAACTTCAGATGAATTTTT

479 ACAAGTTACACAGATTGATACTGTTTGCTTACAATTGCCTATTACAACT TGCTATAAAAA 601 ACAAGTTACACAGATTGATACTGTTTGCTTACAATTGCCTATTACAACTTGCTATAAAAA

539 GTTGGTACAGATGATCTGCACTGTCAAGTAAACTACAGTTAGGAATCCTCAAAGATTGGT

661 GTTGGTACAGATGATCTGCACT GTCAAGTAAACTACAGTTAGGAATCCT CAAAGATTGGT

599 TTGTTTGTTTTTAACTGTAGTTCCAGTATTATAT GATCACTATCGATTTCCTGGAGAGTT

721 TTGTTTGTTTTTAACTGTAGTTCCAGTATTATATGATCACTATCGATTTCCTGGAGAGTT

658 780

659 TTGTAATCTGAATTCTTTATGTATATTCCTAGCTATATTTCATACAAAGTGTTTTAAGAG 781 TTGTAATCTGAATTCTTTATGTATATTCCTAGCTATATTTCATACAAAGTGTTTTAAGAG

719 TGGAGAGTCAATTAAACACCTTTACTCTTAGGAATATAGATTCGGCAGCCTTCAGTGAAT

779 ATTGGTTTTTTCCCTTTGGTATGTCAATAAAAGTTTATCCATGTGTCAGAAAAAAAAAAA 839 901 ATTGGTTTTTTCCCTTTGGTATGTCAATAAAAGTTTATCCATGTGTCAGAAAAAAAAAAA 961

1 MASASCSPGGALASPEPGRKILPRMISHSELRKLFYSADAVCFDVDSTVISEEGIGCFHW

1 MASASCSPGGALASPEPGRKILPRMISHSELRKLFYSADAVCFDVDSTVISEEGIGRSLA
FIGURE 5 | (A) mRNA homology of splicing variants of PSPHL PSPHL_A mRNA sequence ( $839 \mathrm{bp}$ ) is $100 \%$ match to the PSPH homolog C09 gene (503 bp) and L-3-phosphoserine-phosphatase homolog (839 bp; AJ001612).
(B) Protein homology of splicing variants of PSPHL PSPHL_B has a novel spliced 122 bp sequence in the middle of PSPHL_A form alters the reading frame and utilizes a different stop codon. aa and 91 aa. We therefore examined whether the PSPHL transcript was incompletely cloned. We determined the expression of PSPHL in a panel of endometrial cancer cell lines using quantitative PCR and then probed RNA from some of these using a cDNA probe specific to PSPHL to determine the size of the PSPHL message. In cell lines expressing the transcript we noted a message that was 
less than $1 \mathrm{~kb}$ (Figure 6) suggesting that longer forms of PSPHL were not expressed in these endometrial cancers and missing from databases. We next performed $5^{\prime}$ and $3^{\prime}$ prime rapid amplification of cDNA ends (RACE) on a cDNA sample prepared from a pool of uterine carcinomas from African-Americans. Resultant clones
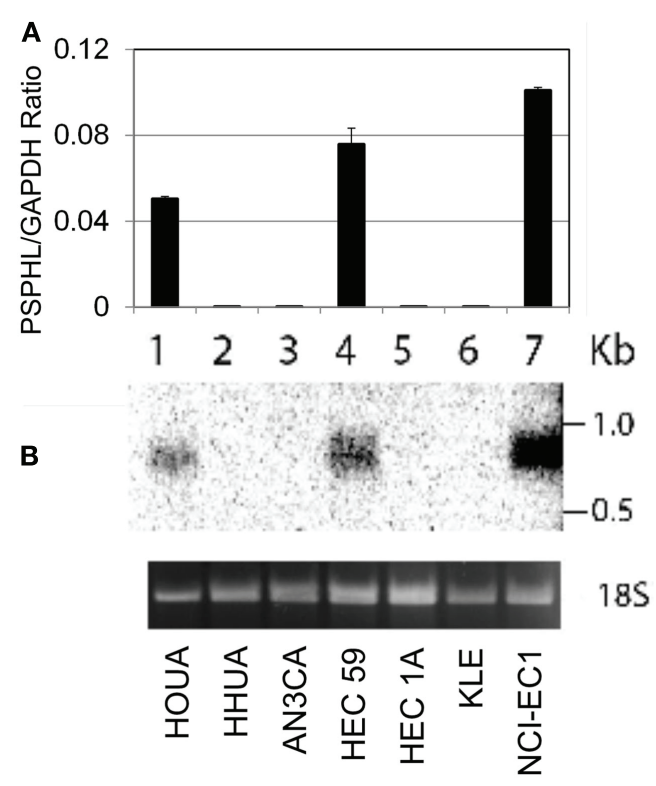

$18 \mathrm{~S}$

FIGURE 6 | (A) Expression levels of PSPHL in uterus cancer cell lines using quantitative PCR. (B) Northern blot analysis using PSPHL specific probes, (1) HOUA (2) HHUA (3) AN3CA (4) HEC 59 (5) HEC 1A (6) KLE (7) NCI-EC1. Ethidium Bromide stain of the $18 \mathrm{~s}$ subunit for loading control. were sequenced and compared to the deposited concensus PSPHL sequence in NCBI. There were two forms of PSPHL identified one of which matched to the consensus sequence. However the second matched the form we identified from ORF specific PCR and has not been described, which we designated form B. Our data indicate that two spliced forms of PSPHL were expressed in these cancers. The length of message on Northern analysis and cloned transcripts are consistent with two forms expressed in these tissues. A diagram of the PSPHL locus and predicted peptides is shown in Figure 5.

\section{PSPHL EXPRESSION IN NORMAL AND TUMOR TISSUES}

We further explored the expression of PSPHL in additional human tissue samples. We wished to determine if PSPHL was expressed in normal endometrium or if there was evidence that supported whether this transcript is differentially expressed in cancers from African-Americans. We examined PSPHL expression in a set of 13 tissue specimens of normal endometrium with seven being from AA and six from CA. Expression of PSPHL was more prominent in the AA specimens (Figure 7). We also found that PSPHL form $\mathrm{B}$ was expressed more frequently in AA tissues (Figure 7). To further examine PSPHL expression we examined normal and cancer tissues from the ovary. We examined a set of stage IIIc serous ovarian cancers and again noted a race difference (Figure 8). Similar to data from the endometrium, normal ovary tissue from AA displayed an elevated level of the transcript for PSPHL whereas there was no significant difference in levels of PSPH (Figure 8), with isoform B being elevated in AA (Figure 8). We next confirmed in a public array set of 39 serous ovarian cancers the expression of PSPHL preferentially in AA cancers compared to CA and no change in $P S P H$ (Figure 9).
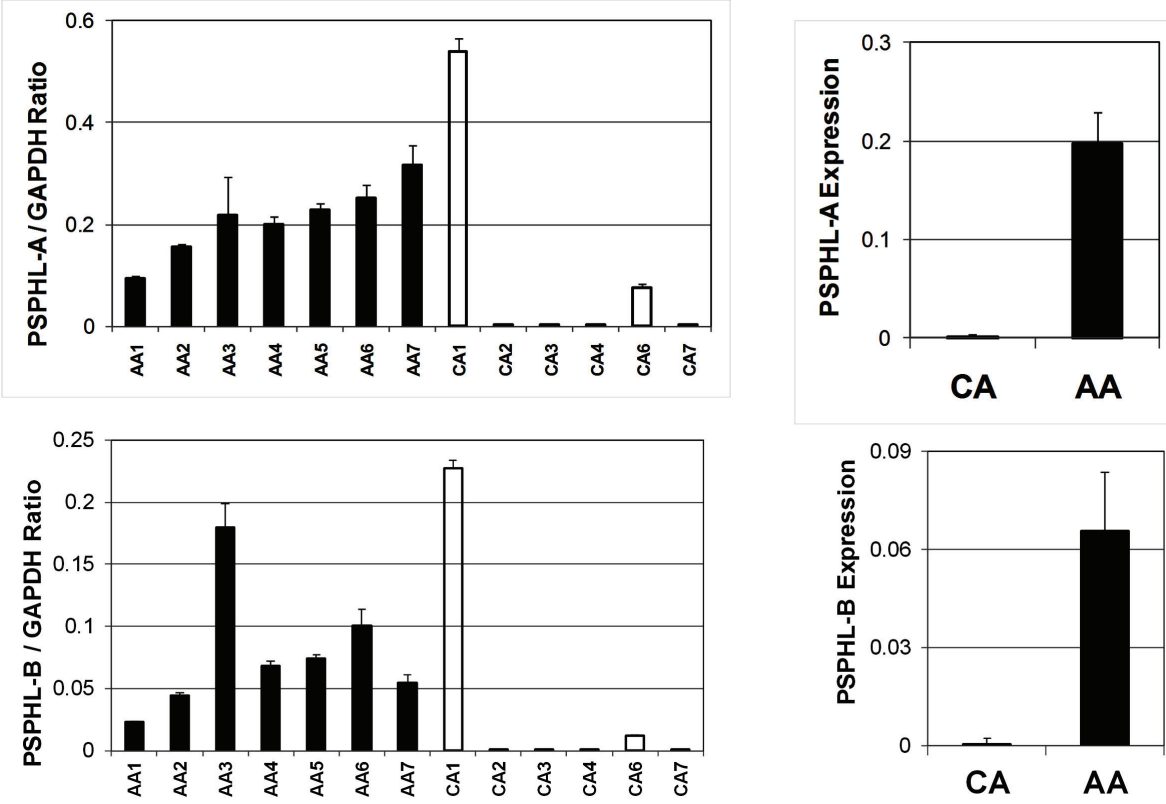

FIGURE 7 | Relative expression levels of PSPHL-A (top) and PSPHL-B (bottom) in normal endometrium for Caucasian and African-American women. Left: individual cases; Right: averages. PSPHLA: AA/CA ratio $=279, p=0.007 ;$ PSPH:L-B AA/CA ratio $=146, p=0.0065$. 
A

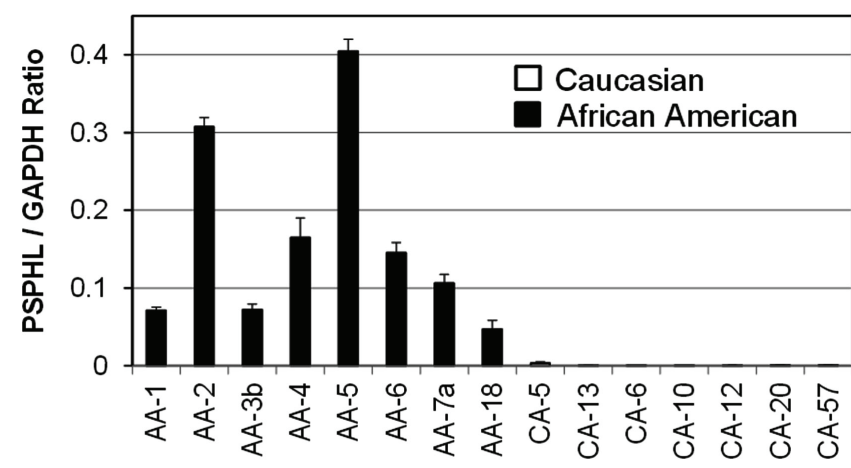

B

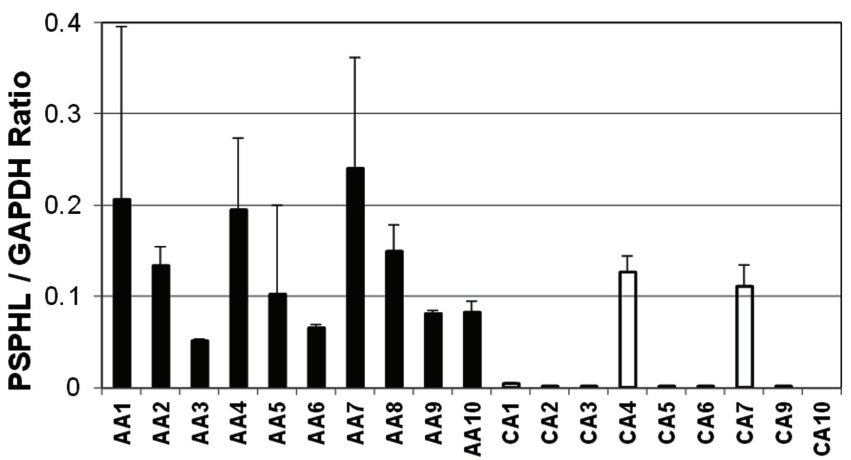

C
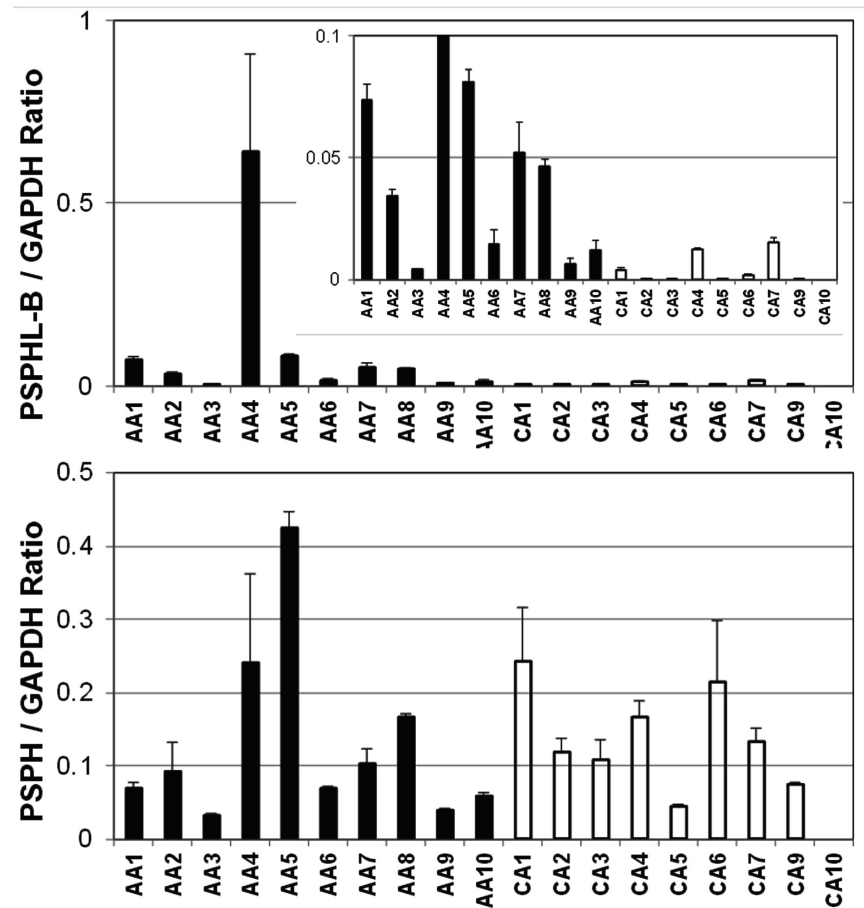
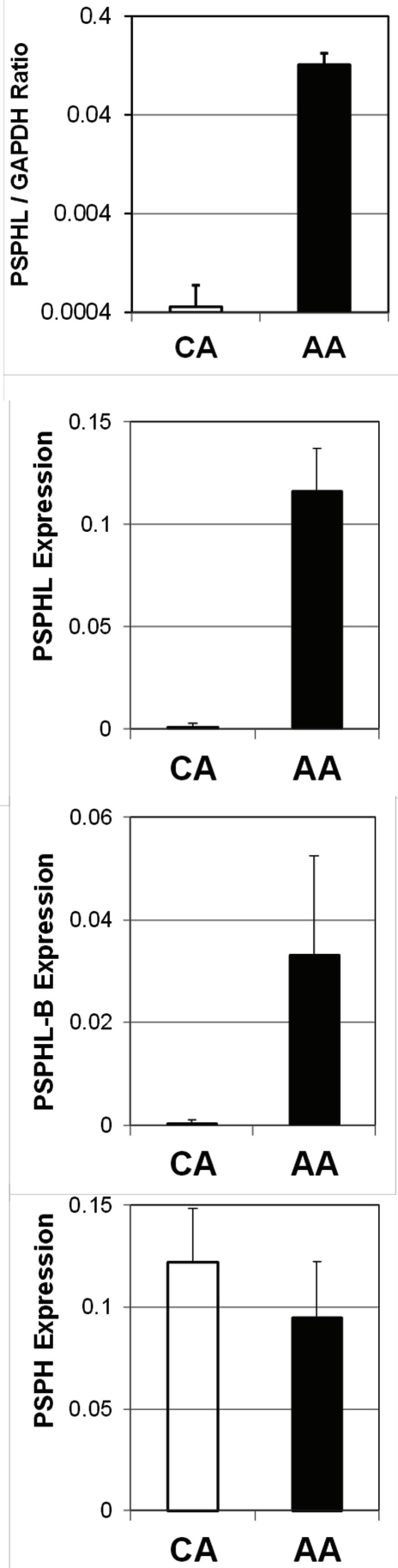

FIGURE 8 | TaqMan validation of expression levels for Caucasian (CA) and African-American (AA) women: (A) PSPHL-A in ovarian cancers, (B) PSPHL-A in normal ovary, (C) PSPHL-B isoform in normal ovary and (D) PSPH in normal ovary. Left Individual cases

\section{PSPHL PROTEIN}

Phosphoserine phosphatase (PSPH) is a 225 amino acid enzyme responsible for catalyzing the last step in the biosynthesis of serine
Right: geometric averages. Error bars indicate standard error. The inset of (C) shows ratios in expanded scale. AA/CA ratios: $A=233 . B=139$. $C=92, D=0.78 ; p$-values $A=1.24 \times 10^{-7} ; B=0.00075 ; C=0.001$; $D=0.46$.

from carbohydrates. The two transcripts correlating to putative phosphoserine phosphatase like protein (PSPHL) could encode a 72 or 91 amino acid homolog of PSPH. However, no evidence 


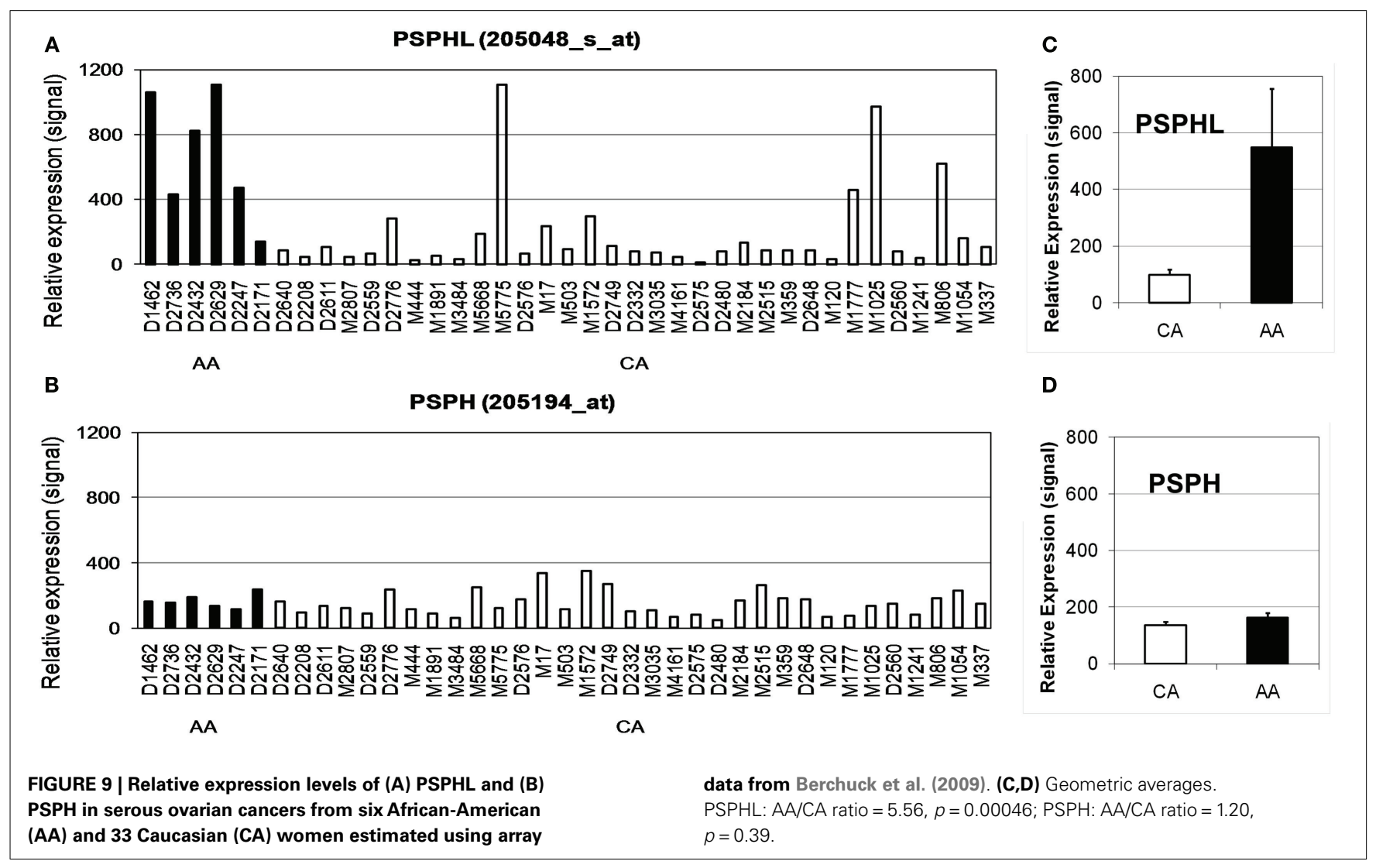

exists to date demonstrating a protein product from $P S P H L$. We cloned and expressed a tagged version of PSPHL in E. coli and a total lysate resolved by $1 \mathrm{D}$ PAGE and Coomassie staining revealed evidence of PSPHL at the predicted mass of $7.8 \mathrm{kDa}$ (not shown). The prominent protein band was excised, in-gel digested with trypsin and peptides and analyzed by nanoflow reversed-phase liquid chromatography (nRPLC) coupled online with tandem mass spectrometry. The collision-induced dissociation spectra resulting from this analysis were searched using SEQUEST (ThermoFisher Scientific) against the human UNIPROT proteome database. The results confirmed the presence of a number of PSPHL peptides confirming the presence of the PSPHL gene product by mass spectrometry. Shown in Figure 10 is the CID spectrum from a unique peptide from PSPHL (MISHSELR), which unambiguously displays prominent fragment ions from the core sequence of the peptide. We utilized this methodology in attempts to detect the PSPHL gene product in human endometrial cancer cells. We examined cell lines with negative and positive endogenous PSPHL transcript expression. We did not identify any PSPHL peptides in either sample supporting the conclusion that the endogenous PSPHL is not found at the protein level (Figure 11). Furthermore it is notable that there exists no evidence in the literature that the PSHPL gene encodes for a functional protein product. Further, this gene has been annotated as likely being a pseudogene by the European Bioinformatics Institute ${ }^{1}$.

\footnotetext{
${ }^{1}$ http://www.uniprot.org/uniprot/O15172
}

\section{EXPRESSION RELATED TO OUTCOME IN GYNECOLOGIC CANCER}

African-Americans exhibit significant health disparities compared to Caucasians in the United States. We examined two array data sets of endometrial and ovarian cancer to determine whether there were differences in expression of PSPHL related to outcome. First we examined a set of 131 uterine cancers for which we knew race and outcome and that had been previously arrayed on Affymetrix chips. These data include 26 AA cases with 3 deaths and $105 \mathrm{CA}$ cases with 10 deaths in the study period of about 16 years between 1989 and 2007. However, we noted few deaths overall in this group of cancers and even fewer among African-Americans. The small number of events limited the strength of survival analysis. We examined the effect of race in this dataset and noted that Caucasians actually survived better (Figures 12A,D) though the result is not statistically significant. We confirmed higher expression of PSPHL in African-Americans cancers in this data set regardless of histologic type (Figures 13 and 14). However, when we examined the overall association of PSPHL expression in this dataset we found there was no difference in survival between high expression and low expression of PSPHL or PSPH (Figures 12B,C).

We next examined a data set of short- and long-term survivors of advanced serous carcinoma of the ovary. We confirmed the higher expression of PSPHL in African-Americans' cancers but again noted an insignificant association with outcome (Figures 12E,F). We did note a high level of PSPHL expression in some short-term survivors but the number of cases was small and when the data are entered in a continuous model of survival statistically insignificant (Figure 14). 


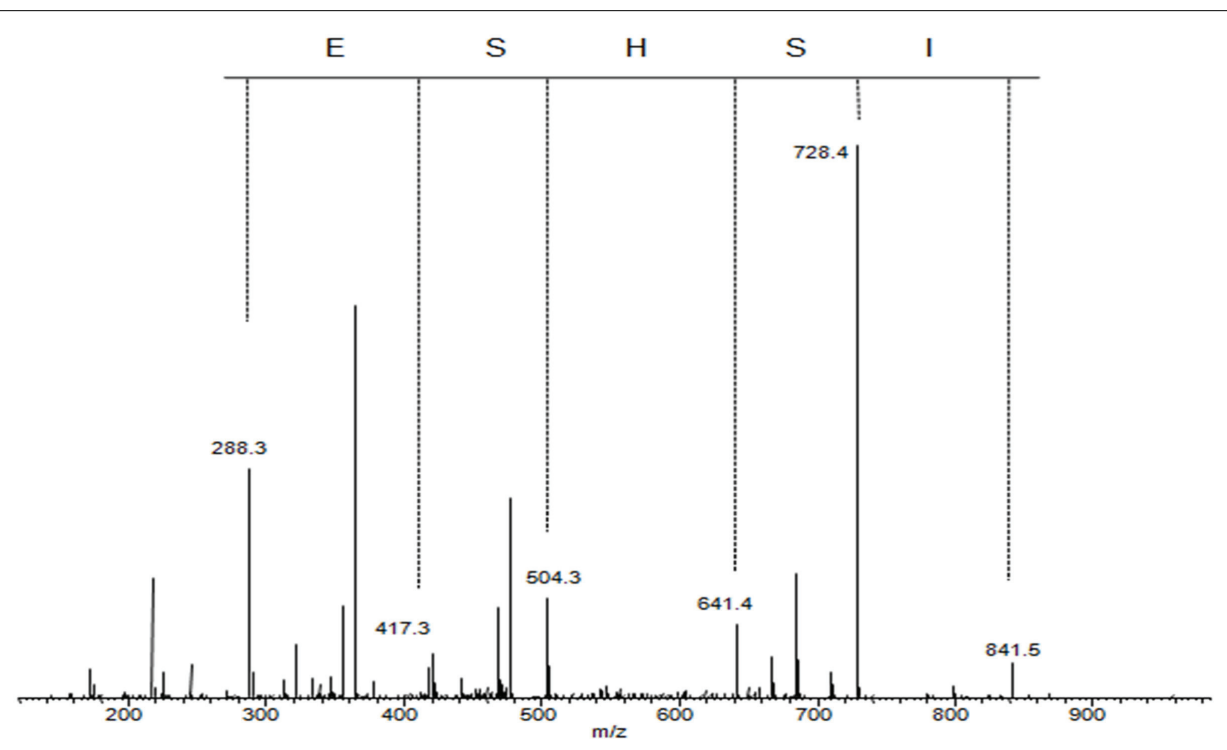

FIGURE 10 | Collision-induced dissociation spectrum of MISHELR, a unique peptide derived from trypsin digestion of PSPHL.

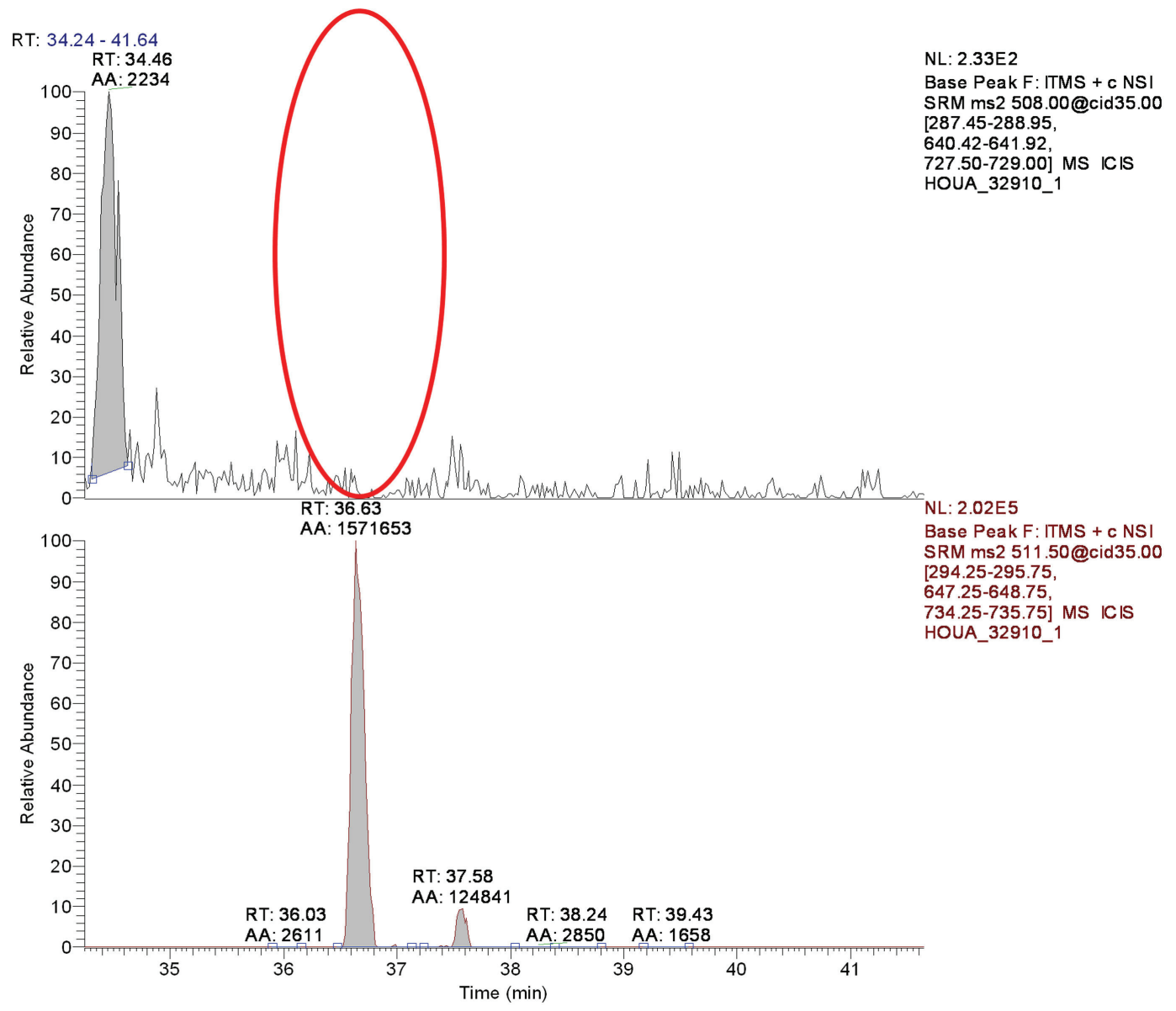

FIGURE 11 | Detection of the diagnostic MISHELR peptide of PSPHL in HOUA endometrial cancer cell lysate. Top panel. Red oval indicates the area where the peptide should be detected, its absence indicating no PSPHL protein in this extract. Bottom shows the recombinant peptide when spiked in the extract. 

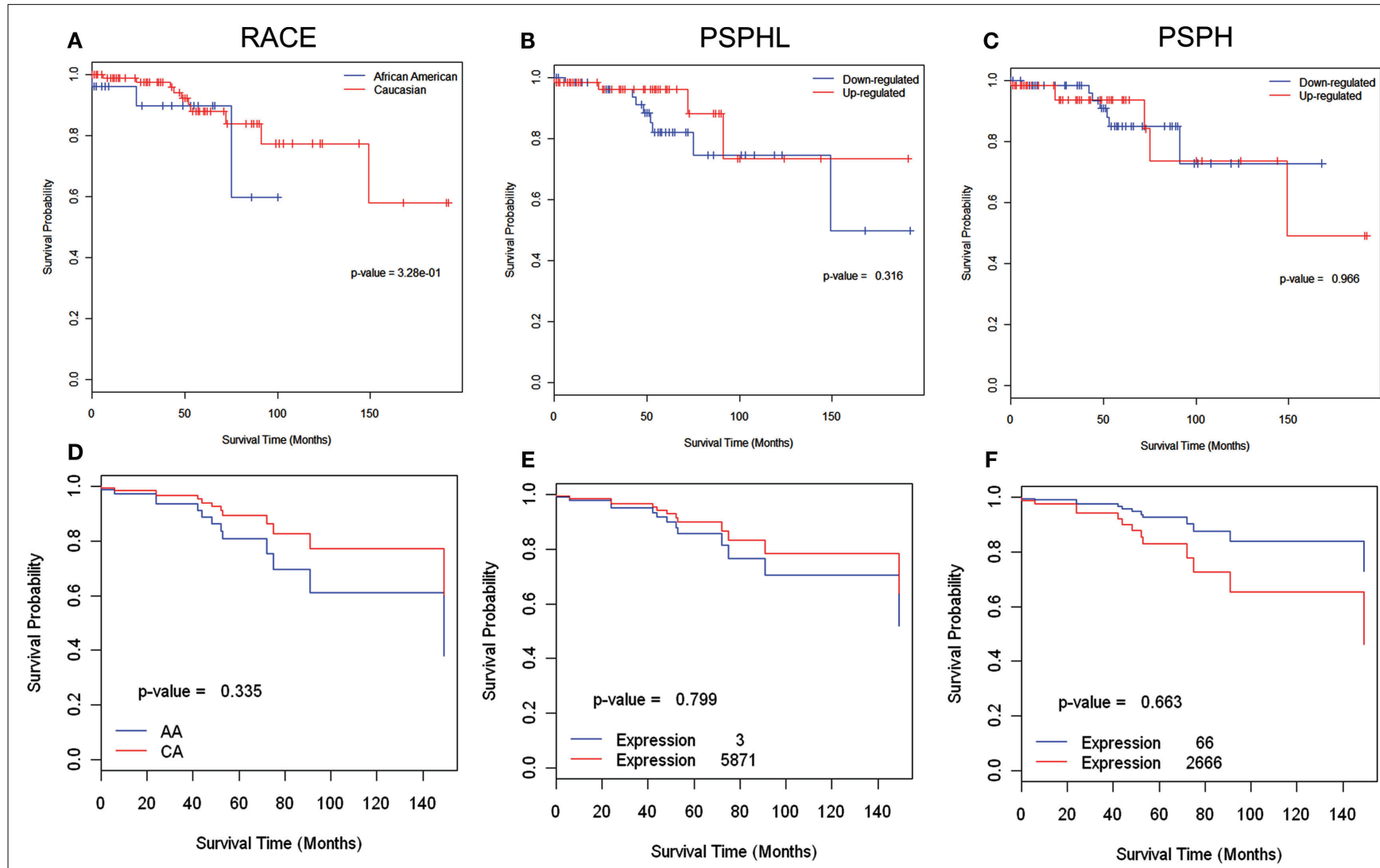

FIGURE 12 | Survival analysis of endometrial cancers using 131 cases (AA-26, CA-105) in which there were I3 (AA-3, CA-10) deaths in the study period of 16 years. Top row Kaplan-Meier curves for (A) Race (B) PSPHL, and (C) PSPH. Up and down-regulations are from median level. The $p$-values are not statistically significant due to small number of events available for the analysis. (D-F) Cox regression analysis of same data showing estimated survival curves: (D) for AA and CA, (E) minimum and maximum signal levels of PSPHL and (F) minimum and maximum signal levels of PSPH.

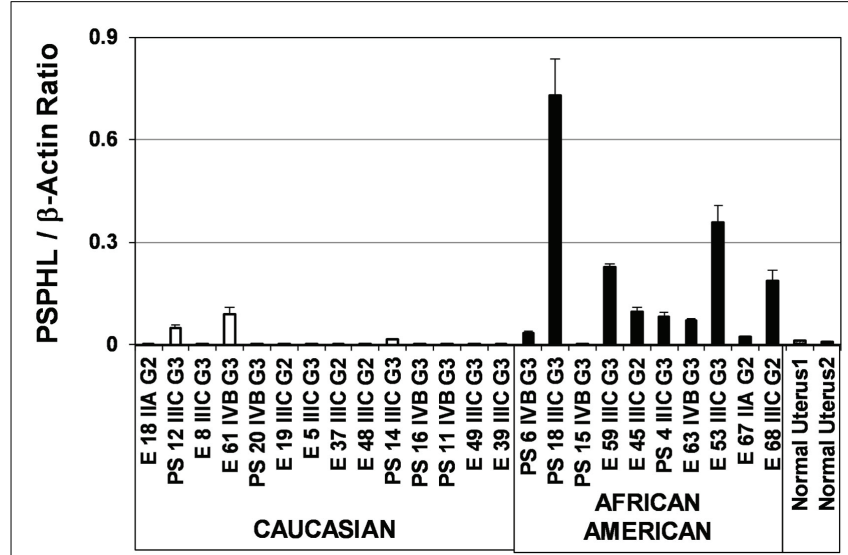

FIGURE 13 | Expression of PSPHL in endometrial cancers from Caucasian. African-American women and normal uterus.

\section{DISCUSSION}

A well recognized racial disparity in incidence and survival outcome exists for endometrial cancer patients in the United States. The racial disparity in outcome is likely multi-factorial. Some reasons include access and utilization of healthcare that may be due to unequal treatment and mistrust of the medical community, as well as differences in cultural acceptance of disease and its fate. However, some authors have suggested that the disparity cannot be explained in total by non-biological factors (Sherman and Devesa, 2003). African-American women tend to be diagnosed with more advanced disease and with adverse histologic types and higher grade, than do Caucasians. These factors are reflective of a more aggressive type of cancer (Steinhorn et al., 1986). Although the endometrial cancers diagnosed in African-American women are often non-endometrioid and poorly differentiated, survival remains worse for African-Americans in investigations that control for stage, grade, histology, and surgical treatment (Randall and Armstrong, 2003).

Molecular genetic data indicates differences in the kinds of mutations that occur in tumors from each group. Several studies have examined the mutational frequency of genes commonly mutated and involved in the pathogenesis of endometrial cancer (Risinger et al., 1997; Tashiro et al., 1997; Suzuki et al., 1998; Stambolic et al., 2000). In addition to differences in DNA mutations several studies have examined other changes to include microsatellite instability, HER2/NEU over expression, and rDNA methylation (Maxwell et al., 2000; Powell et al., 2002; Santin et al., 


\section{Ovarian Serous Cancers}
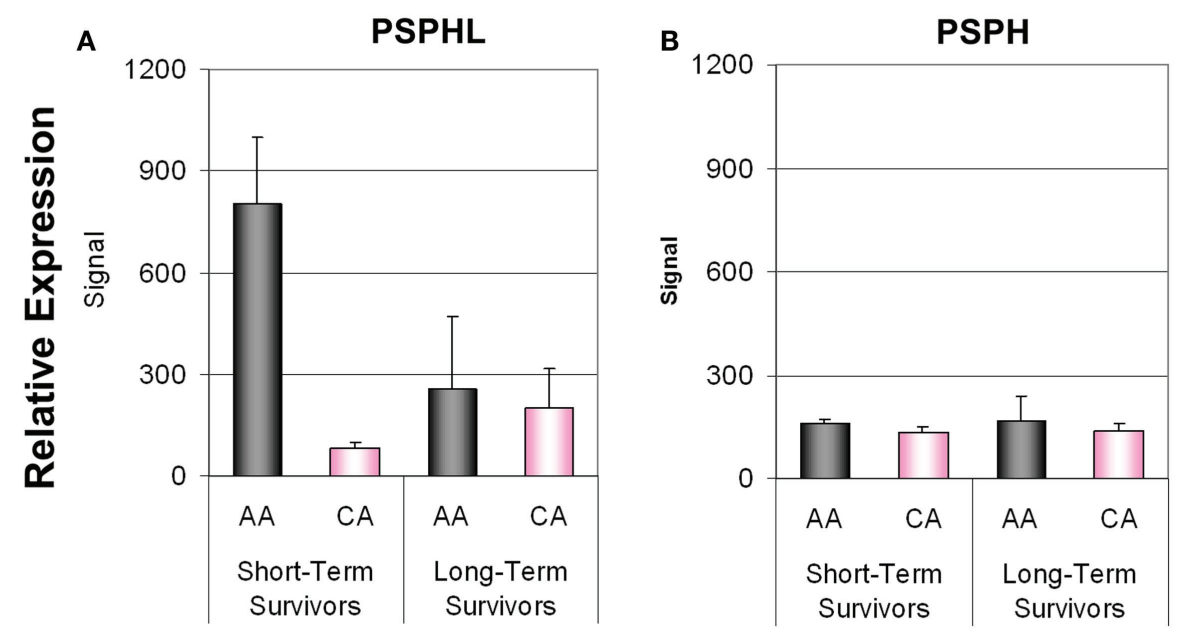

\section{Endometrial Cancers}

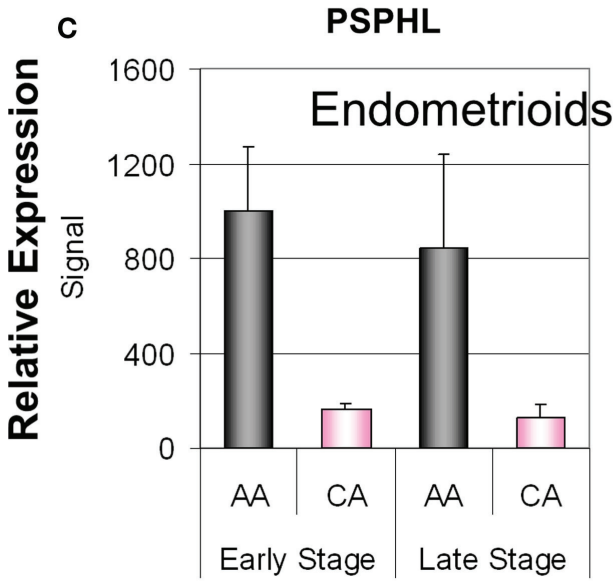

D

PSPHL

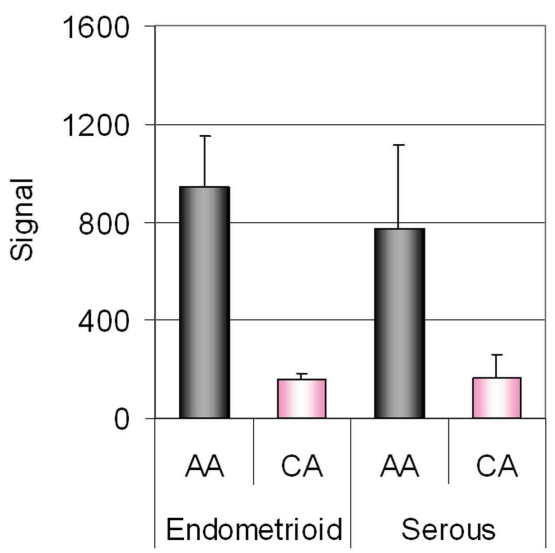

FIGURE 14 | (A) Differential expression of PSPHL in ovarian cancers of short-term survivors. (B) Relative expressions of PSPH in the same ovarian cancers (data from Berchuck et al., 2009). Differential expression of PSPH in

2005). Based in part on these observations Ferguson et al. (2006) examined the gene expression from African-American and Caucasian women and found few statistically differentially expressed genes. We performed a similar type study which is described in this manuscript and found a few more statistically significant genes but largely confirm that underlying expression due to the patients race was not apparent in global expression. We did identify a set of genes that were statistically significant and might represent candidates for evaluation on racial disparity of this disease. However we chose to focus most of our validation efforts on the single probe set that was significantly changed in both sets of data and represented not only robust statistical significance but a significant fold change in expression as well. Interestingly the probe set targeted a poorly described transcript with homology to PSPH. PSPH, Phosphoserine phosphatase (PSP) normally functions in the conversion of L-phosphoserine to L-serine. Serine is one of endometrial cancers: (C) by stage and (D) by histologic type. AA, African-American; CA, Caucasian; Signal, geometric average signal from Affymetrix micro arrays.

the amino acids necessary for protein and nucleotide synthesis. Recently several reports implicate PSPH as a stem cell marker (Skalnikova et al., 2008). Furthermore PSPH has been identified as a gene up-regulated in gastric micrometastasis (Shimomura et al., 2004). Recently PSPH has been suggested to also be involved in cell to cell signaling. However we noted an inability to validate our array finding using a PSPH specific TaqMan assay. We explored the reasons for this and noted that the Affymetrix probes were more likely targeting the poorly described C09 or PSPHL gene also located on chromosome 7 . We were able to confirm the original array findings when we used a probe set specific to PSPHL and not PSPH.

We noted no functional data in the literature for PSPHL and were uncertain to the completeness of the annotation. No protein has been identified for PSPHL and its function as a non-coding RNA or as a protein is unknown. Although it contains a region 
of exact homology with PSPH the predicted peptide from the described gene would encode a very small protein. We further characterized the PSPHL transcript by performing Northern analysis and found that PSPHL specific probe targeted a roughly $900 \mathrm{bp}$ message suggesting that there were not larger PSPHL transcripts. When we performed PCR on the predicted ORF of PSPHL we noted two distinct bands. We cloned these and found an alternative isoform of PSPHL that adds an additional exon and at that point also changes the open reading frame. We performed $5^{\prime}$ and $3^{\prime}$ RACE and were able to isolate PCR clones representing only these two splice forms of PSPHL that were predicted to produce 72 or $91 \mathrm{kDa}$ peptides.

Recently Wei et al. (2011) examined the gene expression from endothelial cells from African-Americans and Caucasians also in an effort to identify underlying gene expression differences related to health disparity. They too noted increased PSPHL expression in endothelial cells in African-Americans and the mis-annotated PSPH probe set. Furthermore they examined the effect of PSPHL expression on its enzymatic L-serine phosphatase activity and found the PSPHL was enzymatically inactive. Additional studies by them indicated that PSPHL did not interfere with normal PSPH activity.

Many cancer sites exhibit racial disparities in outcome and incidence. Prostate and breast cancers cancer disproportionaltely affects AA. Similar expression array studies have been performed for prostate and breast cancers. Wallace et al., identified PSPHL as one of the most racially differentially expressed genes in prostate cancers. Expression was higher in AA men and along with CRYBB2 was an accurate two gene classifier for AA status in prostate cancer tissue but not corresponding normals (Wallace et al., 2008). Similarly this same group identified PSPHL over expression in AA women compared to CA women's breast cancers and surrounding stroma (Martin et al., 2009). Again PSPHL and CRYBB2 were demonstrated to be an accurate two gene classifier for AA status in tumors. PSPHL was one of five genes necessary to classify the stroma of these patients.

Importantly we examined whether PSPHL expression was a tumor derived characteristic or whether the expression was present prior to neoplastic transforming processes. In two different tissue types uterus and ovary PSPHL was expressed more often in both normal and malignant tissue from African-Americans. Indicating that PSPHL expression is not solely a tumor derived characteristic. This is also supported by the findings in breast, prostate, and endothelial cells (Wallace et al., 2008; Martin et al., 2009; Wei et al., 2011). It also suggests that if PSPHL plays any role in the racial disparity of endometrial cancer it could affect numerous processes. Raising the questions of whether presence of PSPHL actually serve to protect endometrial transformation in African-Americans where the disease is less prevalent? Or does it affect some biologic function that could adversely affect outcome such as drug response, invasion, metastatic spread? Or is PSPHL expression just reflective of an underlying racial difference in gene expression and have no relation to endometrial cancer. In this regard we like others could not find evidence for a PSPHL peptide in human cells despite using sophisticated proteomic methods on cells highly expressing the PSPHL transcript. It is likely if PSPHL has function in cells it is occurring through an RNA mediated mechanism. Recently functional roles for LncRNAs have come to light (Prensner and Chinnaiyan, 2011). The abundance of mRNAs may also regulate other genes by a ceRNA modulation of miRNA levels at their 3' UTRs (Poliseno et al., 2010; Salmena et al., 2011; Tay et al., 2011). It is clear that future studies are required to assess the biologic function if any of this transcript.

We did examine two gene expression array datasets with clinical survival endpoints and designated tissues by race to gain insight on whether PSPHL expression is related to survival. We confirmed the preferential expression of PSPHL in African-Americans as compared to Caucasians cancers in both sets one endometrial and one ovarian. As we found throughout our studies a significant number of cancers from Caucasian do express PSPHL. This fact allows us to track survival related to the genes expression rather than using its expression as a racial surrogate. In these analyses we had access to a dataset of 133 endometrial cancers of which only 13 expired. We did not see any association with survival in this set perhaps reflecting the overall good survival in this data set. It will be important to analyze the relevance of PSPHL in larger datasets with more African-Americans and a greater number of adverse survival events. Similarly we noted a non-significant association with survival in serous ovarian cancers a tumor type generally not associated with a racial disparity in outcome. Interestingly we noted that among the short-term survivors in a large set of advanced ovarian cancer that PSPHL was highly expressed in AA tumors although this data was generated from only a few individuals.

In summary we identified that the PSPHL transcript is differentially expressed in endometrial cancers from African-Americans and Caucasians. This tumor type occurs more frequently in Caucasians but is more deadly in African-Americans. PSPHL was also preferentially expressed in normal endometrium of AfricanAmericans as well as normal and malignant ovary tissues. PSPHL may ultimately be found to serve as a prognostic marker for these diseases and could function in numerous cellular processes to include either tumor suppression or by affecting biologic and other treatment events related to outcome.

\section{MATERIALS AND METHODS \\ PSPHL TRANSCRIPT CHARACTERIZATION}

Transcript size was determined by Northern blot of endometrial cancer cell line total RNAs isolated from tumor biopsies. Transcript specific ${ }^{32} \mathrm{P}$ labeled probes were generated by random priming from inserts excised from sequence verified vector containing the ORF of PSPHL. Hybridized blots were washed at high stringency and exposed to phosphor screens. Phosphor screens were scanned using a Molecular Dynamics STORM (GE Healthcare, UK) phosphorimaging system for quantitative measurements. PCR primers designed to suspected PSPHL ORF were ATGGCCAGCGCCAGCTGC and TTATCCTTGACTTGTTGCCTGATC. Rapid Amplification of cDNA Ends (SMART RACE) was performed according to the manufacturers recommendations and using a nested primer strategy. $5^{\prime}$ and $3^{\prime}$ cDNAs were prepared from a pool of four uterine carcinomas from African-American women. Primers utilized in $5^{\prime}$ RACE were CCAGCAGCTCTACAAAATCAGTG and ATTCTTCCGGTTCTCCCAGCAGC. Primers used for $3^{\prime}$ RACE were ATGGCCAGCGCCAGCTGC (same as ORF above) and 
AAGCTTCTCAATGGCCAGC. PCR products were cloned in pENTR TOPO vectors (Invitrogen) according to manufacturers recommendations. Isolated plasmid DNAs were sequenced with vector based primers M13-F and M13-R.

\section{CELL LINES}

AN3CA, KLE, HEC-1-A were obtained from the American Tissue Type Collection, Rockville Md. HEC-59, HOUA, and HHUA were a kind gift from Hiroyuki Takahashi, Jikei University, Tokyo Japan. NCI-EC1 was developed by the corresponding author at the National Cancer Institute (NCI) from a Stage IIIC endometrioid cancer. Cells were grown in DMEM/F-12 supplemented with $10 \%$ fetal bovine serum and maintained in a humidified $5 \% \mathrm{CO} 2$ incubator.

\section{TISSUE SPECIMENS}

Flash frozen cancer specimens were obtained from patients undergoing surgery for uterine cancer at Duke University Medical Center and were part of a microarray data set previously reported (Maxwell et al., 2005). All tissues were collected under an IRB approved protocol at Duke University Medical Center. Specimens were harvested within $30 \mathrm{~min}$ of specimen removal at the time of surgery. Each uterine tumor was then frozen until the time of the analysis. The set of endometrial cancers selected for this analysis included 10 African-Americans and 14 Caucasians. Race determination of patients reflected self-described racial status. Only specimens from African-Americans for which there were specimens from Caucasians that were matched by stage, grade, and histology were considered for analysis. Tissue specimens were evaluated by H\&E to confirm that all specimens comprising the matched pairs contained at least $50 \%$ or greater cancer cells. During preparation of the specimens for analysis, care was taken to macroscopically dissect the cancer away from any adjacent myometrium. Endometrial, ovarian, and breast samples used for quantitative PCR validation of array data were obtained from the cooperative tissue network, Southeast region. Tumor RNAs used in PSPHL cDNA cloning were obtained from Memorial Health University Medical center under an approved IRB protocol. Tissue samples were subjected to RNA isolation following laser capture of epithelial cell fraction using TRIzol followed by an additional level of purification with the Rneasy Kit (Qiagen, Valencia, CA, USA). The integrity of each of the RNA samples was confirmed using denaturing gel electrophoresis.

\section{GENE EXPRESSION ANALYSIS}

The gene expressions were assessed using the Affymetrix human genome HG-U133A and HG-U133B Genechips. Approximately $5 \mu \mathrm{g}$ of total RNA from each sample was labeled using the high yield transcript labeling kit (ENZO) and labeled RNAs were hybridized, washed, and scanned according to the manufacturers specifications (Affymetrix Inc., Santa Clara, CA, USA). Affymetrix Microarray Suite 5.0 software (MAS5) was used to estimate transcript signal levels from scanned images by one step Tukey's biweight algorithm. The signals on each array were normalized to a trimmed mean value of 500 excluding the lowest $2 \%$ and highest $2 \%$ of the signals. An Affymetrix probe set representing a unique Gene Bank sequence is referred as a transcript hereafter for convenience. Unsupervised data analysis was performed using Multidimensional scaling where the distance metric was $1-\rho$ ( $\rho$ is correlation coefficient) and Principal Component Analysis (PCA) by minimizing the correlations (using R-Statistical package). About 18,500 transcripts having detection $p$-value $<0.065$ in at least half of the arrays were included in these analyses. Binary class comparison was performed on the different racial groups using BRB Array tools software (BRB Array tools ver. 3.0c, Richard Simon, Amy Peng, Biometric research branch, NCI, $\mathrm{NIH}^{2}$ ). The transcripts having detection $p$-value $>0.065$ in more than $95 \%$ of the arrays were eliminated for statistical comparisons of classes. Class comparison using two-sample $t$-tests between AA and CA indicated 341 differentially expressed transcripts at $p<0.005$. The significance of finding these 341 transcripts was estimated by a global test using random permutations of class labels (BRB Array Tools) which gave a $p$-value of 0.061 . This lack of global differential expression likely arises from variations in clinical factors. In order to account for variations in histology, stage and grade, we considered matched-pair analysis between AA and CA cases. The matching of $10 \mathrm{AA}$ cases with $14 \mathrm{CA}$ cases resulted in 14 pairs in which four AA cases were duplicated. Class comparison of these matched pairs by paired $t$-tests indicated 325 transcripts at $p<0.005$ with a global test $p$-value of 0.045 . There were 66 transcripts altered by twofold among these. Hierarchical clustering of these transcripts was performed on mean centered gene expressions using $1-\rho$ as distance metric and average linkage algorithm (Salmena et al., 2011). The heat map was color-coded using red for high expression and green for low expression compared to the array average in black. All the statistical calculations were performed on the logarithmic values of signals to the base 2. Survival analysis was done using "survival" package contributed to R-project ${ }^{3}$. A uterine cancer data set including 26 AA cases (with three deaths) and 105 CA cases (with 10 deaths) in a study period of about 16 years between 1989 and 2007 was used for KaplanMeier plots and for Cox regression analysis. Log-rank and Wald tests were used to calculate $p$-values of KM and Cox regression analyses respectively. Log-rank test of AA vs. CA cases indicated a $p$-value of 0.33 . Cox proportional hazards regression analysis indicated poor survival of AA cases at Wald test $p$-value of 0.34 . There was no significant difference in KM curves of the cases having PSPHL expression below the median and above the median level.

\section{VALIDATION OF GENE EXPRESSION USING QUANTITATIVE PCR}

The expression of genes chosen for validation were determined by mulitplex PCR using TaqMan ${ }^{\circledR}$ Gene Expression Assays purchased from Applied Biosystems, (Foster City, CA, USA) with endogenous $\beta$-actin as reference. Samples were run on the ABI Prism ${ }^{\circledR} 7700$ Sequence Detection System according to manufacturer's suggested protocols. The relative quantitation was done using the comparative $C_{\mathrm{T}}$ threshold cycle $\left(C_{\mathrm{T}}\right)$ method, and mean of triplicate $C_{\mathrm{T}}$ measurements was calculated for each sample and the ratios to $\beta$ actin were determined. The geometric average of the mean ratios for each racial group was presented with the standard error of mean values as error bars.

\footnotetext{
${ }^{2} \mathrm{http} / / /$ linus.nci.nih.gov/BRB-ArrayTools.html

${ }^{3} \mathrm{http} / / /$ www.r-project.org/index.html
} 


\section{LIOUID CHROMATOGRAPHY-TANDEM MASS SPECTROMETRY}

The prominent gel band at $8 \mathrm{kDa}$ was excised and in-gel digested with trypsin as previously described (Shevchenko et al., 2006). Peptide digests were analyzed in triplicate by reversed-phase liquid chromatography (Ultimate 3000, Dionex Corporation, Sunnyvale, CA, USA) coupled online to a linear ion trap MS (LTQ$\mathrm{XL}$, ThermoFisher Scientific, San Jose, CA, USA) as previously described (Hood et al., 2010). Tandem mass spectra were searched against the UniProt human protein database (10/08 release) from the European Bioinformatics Institute ${ }^{4}$, using SEQUEST

${ }^{4}$ http://www.ebi.ac.uk/integr8

\section{REFERENCES}

Berchuck, A., Iversen, E. S., Luo, J., Clarke, J. P., Horne, H., Levine, D. A., Boyd, J., Alonso, M. A., Secord, A. A., Bernardini, M. Q., Barnett, J. C., Boren, T., Murphy, S. K., Dressman, H. K., Marks, J. R., and Lancaster, J. M. (2009). Microarray analysis of early stage serous ovarian cancers shows profiles predictive of favorable outcome. Clin. Cancer Res. 15, 2448-2455.

Ferguson, S. E., Olshen, A. B., Levine, D. A., Viale, A., Barakat, R. R., and Boyd, J. (2006). Molecular profiling of endometrial cancers from AfricanAmerican and Caucasian women. Gynecol. Oncol. 101, 209-213.

Ghafoor, A., Jemal, A., Cokkinides, V., Cardinez, C., Murray, T., Samuels, A., and Thun, M. J. (2002). Cancer statistics for African Americans. $C A$ Cancer J. Clin. 52, 326-341.

Hood, B. L., Grahovac, J., Flint, M. S., Sun, M., Charro, N., Becker, D., Wells, A., and Conrads, T. P. (2010). Proteomic analysis of laser micro dissected melanoma cells from skin organ cultures. J. Proteome Res. 9, 3656-3663.

Hicks, M. L., Phillips, J. L., Parham, G., Andrews, N., Jones, W. B., Shingleton, H. M., and Menck, H. R. (1998). The national cancer data base report on endometrial carcinoma in African-American women. Cancer 83, 2629-2637.

Kohler, M. F., Carney, P., Dodge, R., Soper, J. T., Clarke-Pearson, D. L., Marks, J. R., and Berchuck, A. (1996). p53 Over expression in advanced-stage endometrial adenocarcinoma. Am. J. Obstet. Gynecol. $175,1246-1252$.

Martin, D. N., Boersma, B. J., Yi, M., Reimers, M., Howe, T. M., Yfantis, H. G., Tsai, Y. C., Williams, E. H., Lee, D. H., Stephens, R. M., Weissman, A. M., and Ambs, S. (2009). Differences in the tumor microenvironment between African-American and European-American breast cancer patients. PLoS ONE 4:e4531. doi:10.1371/journal.pone.0004531

Maxwell, G. L., Chandramouli, G. V., Dainty, L., Litzi, T. J., Berchuck, A., Barrett, J. C., and Risinger, J. I. (2005). Microarray analysis of endometrial carcinomas and mixed Mullerian tumors reveals distinct gene expression profiles associated uterine cancer. Clin. Cancer Res. 11, 4056-4066.

Maxwell, G. L., and Risinger, J. I. (2006). Racial disparities research: it's not just black and white. Gynecol. Oncol. 101, 194-197.

Maxwell, G. L., Risinger, J. I., Hayes, K. A., Alvarez, A. A., Dodge, R. K., Barrett, J. C., and Berchuck, A. (2000). Racial disparity in the frequency of PTEN mutations, but not microsatellite instability, in advanced endometrial cancers. Clin. Cancer Res. 6, 2999-3005.

Morrison, C., Miecznikowski, J., Darcy, K. M., Dolce, J. M., Kandel, E., Erwin, D. O., Liu, S., Shepherd, L., Cohn, D., McMeekin, D. S., Block, A. W., Nowak, N. J., and Maxwell, L. (2010). A GOG 210 aCGH study of gain at 1q23 in endometrioid endometrial cancer in the context of racial disparity and outcome. Genes Chromosomes Cancer 49, 791-802.

Planitzer, S. A., Machl, A. W., Rueckels, M., and Kubbies, M. (1998). Identification of a novel c-DNA over expressed in Fanconi's anemia fibroblasts partially homologous to a putative L-3-phosphoserinephosphatase. Gene 210, 297-306.

Poliseno, L., Salmena, L., Zhang, J., Carver, B., Haveman, W. J., coding-independent function of gene and pseudogene mRNAs regulates tumour biology. Nature 465, 1033-1038.

Powell, M. A., Mutch, D. G., Rader, J. S., Herzog, T. J., Huang, T. H., and with different histologic types of and Pandolfi, P. P. (2010). A

(ThermoFisher Scientific). Additionally, peptides were searched for methionine oxidation with a mass addition of 15.9949 Da. Peptides were considered legitimately identified if they met specific charge state and proteolytic cleavage-dependent cross correlation scores of 1.9 for $[\mathrm{M}+\mathrm{H}]^{1+}, 2.2$ for $[\mathrm{M}+2 \mathrm{H}]^{2+}$, and 3.5 for $[\mathrm{M}+3 \mathrm{H}]^{3+}$, and a minimum delta correlation of 0.08 .

\section{SUPPLEMENTARY MATERIAL}

The Supplementary Material for this article can be found online at http://www.frontiersin.org/Women's_Cancer/10.3389/ fonc.2012.00065/abstract

Goodfellow, P. J. (2002). Ribosomal DNA methylation in patients with endometrial carcinoma: an independent prognostic marker. Cancer 94, 2941-2952.

Prensner, J. R., and Chinnaiyan, A. M. (2011). The emergence of lncRNAs in cancer biology. Cancer Discov. 1, 391-407.

Randall, T. C., and Armstrong, K. (2003). Differences in treatment and outcome between African-American and white women with endometrial cancer. J. Clin. Oncol. 21, 4200-4206.

Risinger, J. I., Hayes, A. K., Berchuck, A., and Barrett, J. C. (1997). PTEN/MMAC1 mutations in endometrial cancers. Cancer Res. 57, 4736-4738.

Risinger, J. I., Maxwell, G. L., Chandramouli, G. V., Aprelikova, O., Litzi, T., Umar, A., Berchuck, A., and Barrett, J. C. (2005). Gene expression profiling of microsatellite unstable and microsatellite stable endometrial cancers indicates distinct pathways of aberrant signaling. Cancer Res. 65, 5031-5037.

Risinger, J. I., Maxwell, G. L., Chandramouli, G. V., Jazaeri, A., Aprelikova, O., Patterson, T., Berchuck, A., and Barrett, J. C. (2003). Microarray analysis reveals distinct gene expression profiles among different histologic types of endometrial cancer. Cancer Res. 63, 6-11.

Salmena, L., Poliseno, L., Tay, Y., Kats L., and Pandolfi, P. P. (2011). A ceRNA hypothesis: the Rosetta Stone of a hidden RNA language? Cell 146, 353-358.

Santin, A. D., Bellone, S., Siegel, E. R., Palmieri, M., Thomas, M., Cannon, M. J., Kay, H. H., Roman, J. J., Burnett, A., and Pecorelli, S. (2005). Racial differences in the over expression of epidermal growth factor type II receptor (HER2/neu): a major prognostic indicator in uterine serous papillary cancer. Am. J.
Obstet. Gynecol. 192, 813-818.

Sherman, M. E., Carreon, J. D., Lacey, J. V. Jr., and Devesa, S. S. (2005). Impact of hysterectomy on endometrial carcinoma rates in the United States. J. Natl. Cancer Inst. 97, 1700-1702.

Sherman, M. E., and Devesa, S. S. (2003). Analysis of racial differences in incidence, survival, and mortality for malignant tumors of the uterine corpus. Cancer 98, 176-186.

Shimomura, K., Sakakura, C., Takemura, M., Takagi, T., Fukuda, K., Kin, S., Nakase, Y., Miyagawa, K., Ohgaki, M., Fujiyama, J., Fujita, Y., Nakanishi, M., Hagiwara, A., Shirane, M., Okazaki, Y., Hayashizaki, Y., and Yamagishi, H. (2004). Combination of L-3-phosphoserine phosphatase and CEA using real-time RT-PCR improves accuracy in detection of peritoneal micrometastasis of gastric cancer. Anticancer Res. 24, 1113-1120.

Shevchenko, A., Tomas, H., Havlis, J., Olsen, J. V., and Mann, M. (2006). In-gel digestion for mass spectrometric characterization of proteins and proteomes. Nat. Protoc. 1, 2856-2860.

Skalnikova, H., Vodicka, P., Pelech, S., Motlik, J., Gadher, S. J., and Kovarova, H. (2008). Protein signaling pathways in differentiation of neural stem cells. Proteomics 8, 4547-4559.

Stambolic, V., Tsao, M. S., Macpherson, D., Suzuki, A., Chapman, W. B., and Mak, T. W. (2000). High incidence of breast and endometrial neoplasia resembling human Cowden syndrome in pten $+/-$ mice. Cancer Res. 60, 3605-3611.

Steinhorn, S. C., Myers, M. H., Hankey, B. F., and Pelham, V. F. (1986). Factors associated with survival differences between black women and white women with cancer of the uterine corpus. Am. J. Epidemiol. 124, 85-93. 
Suzuki, A., de la Pompa, J. L., Stambolic, V., Elia, A. J., Sasaki, T., del Barco Barrantes, I., Ho, A., Wakeham, A., Itie, A., Khoo, W., Fukumoto, M., and Mak, T. W. (1998). High cancer susceptibility and embryonic lethality associated with mutation of the PTEN tumor suppressor gene in mice. Curr. Biol. 8, 1169-1178.

Tashiro, H., Blazes, M. S., Wu, R., Cho, K. R., Bose, S., Wang, S. I., Li, J., Parsons, R., and Ellenson, L. H. (1997). Mutations in PTEN are frequent in endometrial carcinoma but rare in other common gynecological malignancies. Cancer Res. 57, 3935-3940.

Tay, Y., Kats, L., Salmena, L., Weiss, D., Tan, S. M., Ala, U., Karreth, F., Poliseno, L., Provero, P., Di Cunto, F., Lieberman, J., Rigoutsos, I., and Pandolfi, P. P. (2011).
Coding-independent regulation of the tumor suppressor PTEN by competing endogenous mRNAs. Cell 147, 344-357.

Wallace, T. A., Prueitt, R. L., Yi, M., Howe, T. M., Gillespie, J. W., Yfantis, H. G., Stephens, R. M., Caporaso, N. E., Loffredo, C. A., and Ambs, S. (2008). Tumor immunobiological differences in prostate cancer between African-American and EuropeanAmerican men. Cancer Res. 68, 927-936.

Wei, P., Milbauer, L. C., Enenstein, J., Nguyen, J., Pan, W., and Hebbel, R. P. (2011). Differential endothelial cell gene expression by African Americans versus Caucasian Americans: a possible contribution to health disparity in vascular disease and cancer. BMC Med. 9, 2. doi:10.1186/1741-7015-9-2

Wright, J. D., Fiorelli, J., Schiff, P. B., Burke, W. M., Kansler, A. L., Cohen, C. J., and Herzog, T. J. (2009). Racial disparities for uterine corpus tumors: changes in clinical characteristics and treatment over time. Cancer 115, 1276-1285.

Conflict of Interest Statement: The authors declare that the research was conducted in the absence of any commercial or financial relationships that could be construed as a potential conflict of interest.

Received: 19 March 2012; accepted: 04 June 2012; published online: 04 July 2012. Citation: Allard JE, Chandramouli GVR, Stagliano K, Hood BL, Litzi T, Shoji
Y, Boyd J, Berchuck A, Conrads TP, Maxwell GL and Risinger JI (2012) Analysis of PSPHL as a candidate gene influencing the racial disparity in endometrial cancer. Front. Oncol. 2:65. doi: 10.3389/fonc.2012.00065

This article was submitted to Frontiers in Women's Cancer, a specialty of Frontiers in Oncology.

Copyright (c) 2012 Allard, Chandramouli, Stagliano, Hood, Litzi, Shoji, Boyd, Berchuck, Conrads, Maxwell and Risinger. This is an open-access article distributed under the terms of the Creative Commons Attribution License, which permits use, distribution and reproduction in other forums, provided the original authors and source are credited and subject to any copyright notices concerning any third-party graphics etc. 